\title{
Interfaces in Natural Fibre Composites: effect of surface energy and physical adhesion
}

\author{
C. A. Fuentes ${ }^{\text {a,*}}$, L.Q.N Tran ${ }^{\text {a }}$, C. Dupont-Gillain ${ }^{\text {b }}$, A.W. Van Vuure ${ }^{\text {a }}$, I. Verpoest ${ }^{\text {a }}$ \\ aDepartment of Metallurgy and Materials Engineering (MTM), Katholieke Universiteit Leuven; Leuven, \\ Belgium \\ bInstitute of Condensed Matter and Nanosciences, Université Catholique de Louvain; Louvain-la- \\ Neuve, Belgium \\ *E-mail: Carlos.Fuentes@mtm.kuleuven.be
}




\section{ABSTRACT}

An integrated physical-chemical-mechanical approach was used to study the effect of adhesion on the mechanical strength of composites reinforced with bamboo and coir fibres, and three thermoplastic matrices (Polypropylene (PP), maleic anhydride grafted polypropylene (MAPP), and polyvinylidene fluoride (PVDF). Surface chemical analysis was conducted by X-ray photoelectron spectroscopy, XPS. Surface physical-chemistry was studied by determining surface energy components of fibres and matrices, by performing contact angle measurements with various known test fluids and employing a 3-component model for surface energy. Work of physical adhesion was calculated. Transverse 3-point bending tests on uni-directional composites were conducted to study interface strength and composite strength was determined by longitudinal flexural tests. Results show that increase in physical adhesion can explain the improved interface and longitudinal strength in bambooPVDF and coir-PVDF composites, as compared to the PP composites. For MAPP, no increase in physical adhesion is predicted, but particularly for coir a strong increase in mechanical interface strength and consequently an increase in longitudinal strength was observed, which may be attributed to a chemical adhesion mechanism.

\section{KEYWORDS}

Natural fibre; Bamboo; Coir; Adhesion; Wilhelmy; Wetting; XPS; Molecular Kinetic Theory 


\section{1.- INTRODUCTION}

The use of natural fibres as reinforcement fibre in (polymer) composite materials has attracted interest due to their good and particular mechanical properties, low cost, environmental friendliness and natural abundance, making such fibres possible substitutes to synthetic reinforcing fibre materials (e.g. transport applications [1]).

Among natural fibres, bamboo has one of the most favourable combinations of low density (1.4 $\left.\mathrm{g} / \mathrm{cm}^{3}\right)$ and high stiffness $(\sim 46 \mathrm{GPa})$ and strength $(\sim 800 \mathrm{MPa})[2]$. In terms of specific properties

(normalised to density), it is claimed that they can be compared with glass fibres [3]. In the case of bamboo Guadua angustifolia, it also has a high growth rate, between 11 and $21 \mathrm{~cm}$ per day, reaching its definitive height after 6-7 months. This bamboo is one of the tropical species that has been identified as having a great potential to fix atmospheric carbon dioxide which makes it an effective plant in terms of global warming prevention and a suitable resource for fibre production [2]. Moreover, the use of bamboo Guadua angustifolia fibres as reinforcement for an epoxy matrix has already been reported, reaching $78 \%$ of the theoretical strength $(383 \mathrm{MPa})$ with a flexural strength of $310 \mathrm{MPa}$, and $95 \%$ of the theoretical stiffness (22 GPa) with a measured modulus of $21 \mathrm{GPa}$ [2].

In contrast, coir fibres are not very strong $(\sim 180 \mathrm{MPa})$ and stiff $(\sim 4 \mathrm{GPa})$, but exhibit high strain to failure (approximately 40\%) which may ameliorate toughness when they are used in composites [4]. Additionally, coir fibres have low density $\left(\sim 1.2 \mathrm{~g} / \mathrm{cm}^{3}\right)$, they are cheap, and available in large quantities.

Bonding between the reinforcing fibre and the matrix has a significant effect on the properties of the composite since stress transfer and load distribution efficiency at the interface is determined by the degree of adhesion between the components. However, the hydrophilic nature of natural fibres reduces their potential as reinforcing agents due to low interfacial interactions with certain important hydrophobic thermoplastic matrices, such as polyethylene and polypropylene, leading to a poor interfacial strength $[1,5,6]$. 
The adhesion at the interface can be described as a combination of physical adhesion (related to wettability), chemical bonding, and mechanical interlocking $[7,8]$. When only physical interactions are analyzed, then the interfacial strength of a composite can be related to the surface energies of the fibre and the matrix in a given system. Accordingly, the fibre's surface chemical composition plays a vital role in obtaining a good fibre-matrix interface. Using the experimental data obtained from wetting measurements, fibres and matrices can be examined and matched in terms of their surface energy components.

The effect of physical adhesion on the mechanical properties of a composite structure not only affects the composite strength, but it is also important for moisture resistance and impact behaviour (high adhesion typically leads to lower penetration impact energy, but it is favourable for damage tolerance).

The wettability and compatibility of the fibre and the matrix can be assessed by analysis of their surface energies [4]. This can be done by measuring contact angles, which is a quantitative measure of solid-liquid molecular interactions and thus provides information on the surface energy of solids. The composite interface can also be studied by mechanical tests which can e.g. be performed on bulk laminate composites by using transverse 3 point bending tests on an uni-directional composite.

In this study, systematic experimental results describing the dynamic wetting properties of bamboo and coir fibres were analyzed by applying the molecular-kinetic theory of wetting (MKT). These natural fibres were selected due to fact that the chemical composition of their surfaces is different $[4,9]$. It was thus verified if bamboo and coir fibre surfaces represent well defined systems for wetting analysis, and how interface properties behave according to the natural fibre's surface composition. The fibre-matrix work of adhesion was calculated to predict the physical adhesion and compatibility of the composites. Flexural transverse three-point bending tests on unidirectional composites were performed to determine interfacial strength, to verify the interface compatibility. 


\section{2.- MATERIALS AND METHODS}

\subsection{Materials}

Technical bamboo fibres of the species Guadua angustifolia were extracted from bamboo culms with a newly developed and proprietary extraction process, giving a maximum fibre length between 20 and $35 \mathrm{~cm}$. Bamboo fibres were obtained from well defined locations in Colombia. There was not any retting and chemical treatment applied before extracting the fibres. Vietnamese coir fibres were extracted from the husk shell of coconuts after a retting step was applied [3].

The technical fibres that were used for the MKT fitting process underwent the following preparation procedure: After being selected (by means of an optical microscope), the fibres were cleaned, first with warm water for one hour $\left(90^{\circ} \mathrm{C}\right)$, then wiped with ethanol with a piece of cotton tissue before being dried in a vacuum oven at $80^{\circ} \mathrm{C}$ for one hour. With the aim to smoothen the lignin at the fibre surface, bamboo fibres were also further put in an autoclave under 3 bars of pressure at $150{ }^{\circ} \mathrm{C}$ for one hour (for more details on this procedure see our previous publication [9]).

Polypropylene, $0.3 \%$ maleic anhydride grafted polypropylene and polyvinylidene fluoride (Solef 1008) were obtained from Propex, Dupont, and Solvay respectively in the form of films. These films were washed with a detergent (RBS-35 from Chemical Products) at a concentration of 4\% v/v in water during one hour, and next rinsed in ultrapure water at $90^{\circ} \mathrm{C}$ for one hour.

\subsection{Composite preparation}

Unidirectional composites were prepared by compression moulding of 4 stacks of prepregs consisting of technical fibres, compressed between thermoplastic films. In order to obtain good impregnation, the temperature used was $200^{\circ} \mathrm{C}\left(25^{\circ} \mathrm{C}\right.$ higher than the melting temperature of PVDF, the polymer with the highest melting temperature). Furthermore, a pressure of 20 bars was used, and a short consolidation time of 5 minutes in order to prevent thermal degradation. The natural fibres and prepregs 
were dried overnight at $65^{\circ} \mathrm{C}$ to prevent problems due to moisture. Fibre volume fraction was set at $40 \%$ by weight measurements.

\subsection{Contact angle measurements and wetting analysis}

Advancing and receding contact angles of various test liquids (with different surface energy components) were measured on the polymer films under controlled conditions (temperature of $20^{\circ} \mathrm{C}$ and humidity of $60 \%$ ), with a Krüss K100 tensiometer using the Wilhelmy technique [4,9]. In order to better describe both the low surface energy and the high surface energy components of the analyzed surfaces, the average of the cosines of the advancing $\left(\theta_{a d v}\right)$ and receding $\left(\theta_{\text {rec }}\right)$ angles was used to estimate the cosine of the equilibrium angle $\left(\theta_{0}\right)$, as has been suggested by Andrieu et al. [10], and is shown in Eq.1

$$
\cos \theta_{0}=0.5 \cos \theta_{a d v}+0.5 \cos \theta_{r e c}
$$

The contact angles of the natural fibres were measured in a similar fashion, albeit only advancing angles could be measured so far, due to surface irregularities on the natural fibres making measurements of receding angles almost impossible (due to trapped liquid). To apply surface energy component theories, static equilibrium contact angles are required. First, the molecular kinetic theory (MKT) was applied to obtain the static advancing angles [11]. By using dynamic contact angle measurements with various test fluids on bamboo and coir fibres, and by applying the MKT theory, it is possible to verify the quality of the measurements by monitoring if the expected immersion velocity dependence, reproducibility and stability of the advancing contact angle data are obtained. This would then indicate that the measured contact angles are the true advancing contact angles. More details are shown in our previous studies [4,9].

$$
v=2 K_{0} \lambda \sinh \left[\frac{\gamma_{L V}\left(\cos \theta_{0}-\cos \theta\right)}{2 n k_{B} T}\right]
$$


where $K_{0}$ is the equilibrium molecular jump frequency, $\lambda$ is the distance between two adsorption sites, $\gamma_{L V}$ is the surface tension of the liquid in contact with vapour, $\theta_{0}$ is the advancing static contact angle and $\theta$ is the dynamic contact angle corresponding to measurement at velocity ; $\mathrm{n}$ is the number of adsorption sites per unit area, $k_{B}$ is Boltzmann's constant and $\mathrm{T}$ is the temperature [11].

\subsection{Surface energy analysis}

Surface energies and components of the surface energies of the fibres and matrices can be estimated using the contact angle data of various test liquids on the fibres and matrices. Van Oss, Chaudhury and Good developed a model that considers the acid-base interaction between molecules, dividing the surface energy into a Lifshitz-van der Waals $\left(\gamma^{\mathrm{LW}}\right)$, an acid $\left(\gamma^{+}\right)$and a base $\left(\gamma^{-}\right)$component [12], as is shown in Eq.3.

$$
\gamma_{s}=\gamma_{s}^{L W}+2 \sqrt{\gamma_{s}^{+} \gamma_{s}^{-}}
$$

The calculation of the acid-base surface free energy components was performed by using SurfTen 4.3 software [13].

The work of adhesion, $W_{a}$, can be described as the work required to disjoint a unit area of the solid-liquid interface, thereby creating a unit area of liquid-vacuum and solid-vacuum interface [14]. The work of adhesion is defined in terms of surface free energies by the Dupré equation:

$$
W_{a}=\gamma_{S}+\gamma_{L}-\gamma_{S L}
$$

Where $\gamma_{S}$ is the surface energy of the solid, $\gamma_{L}$ is the surface energy of the liquid or matrix and $\gamma_{S L}$ is the interfacial energy. The latter can be expressed in terms of Lifshitz-van der Waals $\left(\gamma^{\mathrm{LW}}\right)$ and acid-base $\left(\gamma^{\mathrm{AB}}\right)$ components as:

$$
\gamma_{s l}=\gamma_{s}^{L W}+\gamma_{l}^{L W}+2\left[\left(\gamma_{s}^{+} \gamma_{s}^{-}\right)^{1 / 2}+\left(\gamma_{l}^{+} \gamma_{l}^{-}\right)^{1 / 2}-\left(\gamma_{s}^{L W} \gamma_{l}^{L W}\right)^{1 / 2}-\left(\gamma_{s}^{+} \gamma_{l}^{-}\right)^{1 / 2}-\left(\gamma_{s}^{-} \gamma_{l}^{+}\right)^{1 / 2}\right]
$$


2.4 Surface characterization: X-ray photoelectron spectroscopy (XPS)

XPS analyses were performed on a Kratos Axis Ultra spectrometer (Kratos Analytical Manchester - UK) equipped with a monochromatized aluminium X-ray source (powered at $10 \mathrm{~mA}$ and $15 \mathrm{kV}$ ). More information regarding the XPS analysis procedure can be found in [9].

\subsection{Composite flexural testing}

Flexural three point bending tests were performed on a universal testing machine (Instron 4426) based on ASTM D790, both in transverse direction to obtain a value for the interface strength and in longitudinal direction to evaluate composite strength.

\section{3. - RESULTS AND DISCUSSION}

\subsection{Surface analysis}

The decomposed $\mathrm{C} 1 \mathrm{~s}$ spectrum, which corresponds to electrons originating from the 1s orbitals of the carbon atoms at the technical bamboo fibre's surface, is shown in figure 1. The C1s peak intensity at $\sim 285 \mathrm{eV}$ is related to the presence of lignin [15]. Cellulose is ideally devoid of aliphatic carboncarbon bonds (designated as $\mathrm{C} 1$ ) because of its polysaccharide structure; however, milled wood lignin shows $49 \%$ of $\mathrm{C} 1$ type carbon atoms [16]. The bamboo results give an average of $58 \%$ of $\mathrm{C} 1$ type carbon (see table 1), showing that lignin is certainly predominant on the surface of bamboo fibres, among other compounds that contain aliphatic-carbon bonds as well [9]. Lin [17] has studied the lignification process of bamboo stems by observing their cross-sections and it was observed that lignin surrounds the bamboo technical fibre and elementary fibres. Hence, a layer of lignin will remain on the surface of mechanically extracted bamboo fibres, even if the bean-shaped fibre breaks up in various technical fibres upon extraction (see figure 2).

In the coir fibre, the high proportion of $\mathrm{C} 1$ carbon (66.2\%) suggests a combination of hydrocarbon rich waxes and lignin. This is supported by the low proportion of $\mathrm{C} 2-\mathrm{C} 4$ (see figure 1 and 
Table 1). Contrarily to bamboo, coir fibres were removed from the husk shell of coconut from the coconut palm (Cocos nucifera L.), suggesting a surface richer in waxes than lignin.

Table 1 presents the surface components of bamboo and coir fibres detected by XPS, consisting of relative atomic percentages of the elements, the oxygen-carbon ratio and decomposition of the $\mathrm{C} 1 \mathrm{~s}$ peak into four sub-peaks $\mathrm{C} 1-\mathrm{C} 4$, representing: $\mathrm{C}-\mathrm{C}$ linkages of lignin, hemicelluloses and extractives (C1); $\mathrm{OH}$ groups of cellulose, hemicelluloses, lignin and extractives (C2); OCH groups of lignin and $\mathrm{C}-$ $\mathrm{O}-\mathrm{C}$ linkages of extractives $(\mathrm{C} 2) ; \mathrm{C}=\mathrm{O}$ groups in lignin and extractives $(\mathrm{C} 3)$; $\mathrm{O}-\mathrm{C}-\mathrm{O}$ linkages in cellulose and hemicelluloses (C3); $\mathrm{CH} 3 \mathrm{CO}$ groups of hemicelluloses (C4), HOOC groups of hemicelluloses $(\mathrm{C} 4), \mathrm{COO}$ and $\mathrm{COOH}$ groups of extractives $(\mathrm{C} 4)$.

\subsection{Surface energy components and adhesion optimization}

The advancing dynamic contact angles of bamboo and coir fibres in water are velocity dependent as was shown in [4] and [9] by fitting the measured angles with the MKT. This behaviour may ensure that the advancing contact angle of our test liquids on autoclave treated bamboo fibre and coir fibre is stable, and reproducible, allowing meaningful information on interfacial interactions to be deduced. Moreover, the advancing static contact angle of coir and bamboo with different liquids can be obtained by extrapolation of the MKT fitting curve (see figure 3).

The same fitting procedure was applied for diiodomethane and ethylene glycol on coir and bamboo. The results of the advancing static contact angles following the MKT fitting procedure are presented in Table 2. For the case of the matrices, both the advancing $\left(\theta_{a d v}\right)$ and receding $\left(\theta_{\text {rec }}\right)$ angles to estimate the equilibrium angle $\left(\theta_{\text {equ }}\right)$ have been used (Equation 1) in order to most accurately describe the surface energy components of the analyzed surfaces. The latter results are also shown in Table 2. 
The static advancing contact angles that were obtained previously for water, diiodomethane and ethylene glycol on bamboo and coir, can now be used to determine the surface energy of the bamboo (see Table 3), according to the Van Oss, Chaudhury and Good approach [12]. It can be seen that the bamboo fibres' surface is more basic than coir. The Lewis basicity of bamboo is three times higher than for coir while its acidity is about the same as for coir. The latter corresponds well with the XPS results, which suggest a surface richer in waxes for coir and, hence, more hydrophobic and less polar than for bamboo.

For the case of PP and MAPP, we find a deviation in the magnitude of the polar surface energy component, which may be zero since pure PP is a nonpolar polymer. This effect could be related to aging processes or surface contamination [18]. In the same fashion, the determined surface energy of MAPP shows an expected increment of polar components due to the presence of maleic anhydride [18]. However, the determined surface energy of MAPP is not far different from that of PP since grafting of maleic anhydride on PP represents just a very small fraction $(0.3 \mathrm{wt} \%)$. As expected, PVDF possesses higher acidity among the three examined matrices due to the different electro-negativities of carbon, fluorine, and hydrogen. Accordingly, the total surface energy of PVDF is higher than that of PP and MAPP, with a higher polar fraction [19].

The work of adhesion for bamboo and coir as substrates were calculated by using equations 4 and 5 (see table 4). The work of adhesion shows a higher value with PVDF for both bamboo and coir composites; this is a consequence of the higher surface energy of PVDF, and electron donor-acceptor interactions due to the presence of a high acid component in PVDF and a high basic component in coir and particularly bamboo. As expected the results for PP and MAPP present a low work of adhesion due to their low polar content. 


\subsection{Interfacial strength}

The interfacial strength of unidirectional composites measured by 3 point bending in transverse direction to the fibre orientation and the efficiency factor of the longitudinal bending strength are shown in Table 5. The efficiency factor is the ratio of experimental longitudinal strength and the calculated value by following the rule of mixtures (= the theoretical strength). This factor is related to the effectiveness of load transfer between the polymer and the fibre, and it was calculated by considering a tensile strength of $700 \mathrm{MPa}$ for bamboo, $180 \mathrm{MPa}$ for coir, $55 \mathrm{MPa}$ for PVDF, $30 \mathrm{MPa}$ for PP, and 18 MPa for MAPP. The reported tensile strength values for the fibres were measured at our lab, and the values for the polymers were provided by the manufacturer.

In the case of bamboo, as predicted by table 4, the results for PVDF are the best, as can be seen in figure 4, proving that the physical adhesion has really been improved. The interface strength for MAPP is higher than in case of PP. This can be explained by the fact that MAPP is capable of forming covalent bonds with the fibre, while PP can only establish physical adhesion. However, the efficiency factor is almost the same for both systems. As explained before, bamboo technical fibres are covered by a lignin layer, reducing its capability to form covalent bonding with MAPP. The latter is not the case for coir, as will be discussed below.

For coir fibres, there is an improvement in interfacial strength for MAPP and PVDF systems in comparison with PP, as is shown in figure 4. While the work of physical adhesion is similar in both PP and MAPP, the transverse strength is almost 7 times higher, probably due to chemical bonding of the coir surface with MAPP. Surprisingly, the longitudinal strength efficiency of coir-PP is not that low (given the low transverse strength). Perhaps, surface roughness of the fibres ensures a better interface strength in shear. This will be checked in future by performing single fibre pull-out tests. 
For coir-PVDF, also a positive effect of improved physical adhesion on both transverse and longitudinal flexural strength is observed, as compared to PP. The mechanism here seems again dominated by physical adhesion.

\section{CONCLUSIONS}

An integrated physical-chemical analysis was conducted to correctly characterize the surface composition of bamboo and coir technical fibres. The results of the characterization of fibre surface chemistry using XPS are consistent to these of wetting measurements, showing the intermediate polarity of both bamboo and coir surfaces with a somewhat higher polarity of the bamboo.

The interfacial adhesion of natural fibre composites was examined by using transverse 3 point bending tests. The results are in agreement with work of adhesion and surface energy analysis predictions obtained from contact angle measurements, showing their efficacy for evaluating the compatibility of natural fibres and matrices for making composites, in so far as the mechanism of adhesion involved is dominated by physical adhesion.

PVDF composites present the best results, showing a high work of adhesion, as well as high interfacial strength and a high efficiency factor for longitudinal strength. This is a consequence of strong physical interactions at the interface due to the presence of a high acid component in PVDF and a high basic component on the surface of bamboo and coir fibres.

The adhesion mechanism in the case of MAPP appears dominated by chemical adhesion, to the extent that chemical groups are present on the fibre surface to react with the MA groups of the MAPP, as is apparently more the case for coir than for bamboo. 


\section{ACKNOWLEDGEMENTS}

Our thanks to Gregory Pyka and Lina Osorio for the Micro-CT and the SEM bamboo images respectively (figure 2). We would also like to acknowledge the financial support of K.U. Leuven (SBA and IRO Scholarships), and the Belgian Science Policy Department (BelSPO) for supporting our research. 


\section{BIBLIOGRAPHY}

1. E. Trujillo, L. Osorio, C. Fuentes, A. Van Vuure, I. Verpoest. Bamboo fibre thermoplastic composites for transport applications. Proceedings of the $31^{\text {st }}$ SAMPE Europe International Conference SEICO 10 , (2010) April 12-15; Paris, France.

2. L. Osorio, E. Trujillo, A.W. Van Vuure, I. Verpoest. Journal of Reinforced Plastics and Composites. 30, 396-408 (2011).

3. T. Dieu, N. Liem, T. Mai, N. Tung. SME International Journal Series A. 47, 570-573 (2004).

4. L.Q.N. Tran, C.A. Fuentes, C. Dupont-Gillain, A.W. Van Vuure and I. Verpoest. Colloids and Surfaces A: Physicochemical and Engineering Aspects. 377, 251-260 (2011).

5. P. Wambua, J. Ivens, I. Verpoest. Composites Science and Technology. 63, 1259-1264 (2003).

6. C. Y. Lai, S. M. Sapuan, M. Ahmad, N. Yahya. Polymer-Plastics Technology and Engineering. 44, 619-632 (2005).

7. C. T. Chou, L. S. Penn. The Journal of Adhesion. 36, 2-3 (1991).

8. J. D. Venables. Journal of Materials Science. 19, 2431-2453 (1984).

9. C.A. Fuentes, L.Q.N. Tran, C. Dupont-Gillain, W. Vanderlinden, S. De Feyter, A.W. Van Vuure, I. Verpoest. Colloids and Surfaces A: Physicochemical and Engineering Aspects. 380, 89-99 (2011).

10. C. Andrieu, C. Sykes and F. Brochard. Langmuir. 10, 2077-2080 (1994).

11. T.D. Blake and J.D. Coninck. Adv. Colloid Interface Sci. 96, 21-36 (2002).

12. C.J. Van Oss, R.J. Good, M.K. Chaudhury. Journal of Colloid and Interface Science. 111, 378-390 (1985).

13. C. Della Volpe and S. Siboni. SurfTen 4.3, A program for the calculation of acid-base solid surface free energy components, http://devolmac.ing.unitn.it:8080/ (2004).

14. E. Pisanova, S. Zhandarov, E. Mäder. Composites: Part A. 32, 425-434 (2001).

15. L.S. Johansson, J.M. Campbell, K. Koljonen, P. Stenius. Appl. Surf. Sci. 144, 92-95 (1999).

16. A. Shchukarev, B. Sundberg, E. Mellerowicz and P. Persson. Surf. Interface Anal. 34, 284-288 (2002).

17. J. Lin, X. Hea, Y. Hua, T. Kuanga and R. Ceulemans. Physiol. Plant. 114, 296-302 (2002).

18. I. Aranberri-Askargorta, T. Lampke, A. Bismarck. Journal of Colloid and Interface Science. 263, 580-589 (2003).

19. S. Lee, J-S Park, T.R. Lee. Langmuir. 24, 4817-4826 (2008). 